\title{
Accuracy Assessment of Global and Local Atrophy Measurement Techniques with Realistic Simulated Longitudinal Data
}

\author{
Oscar Camara ${ }^{1}$, Rachael I. Scahill ${ }^{2}$, Julia A. Schnabel ${ }^{1}$, William R. Crum ${ }^{1}$, \\ Gerard R. Ridgway ${ }^{1}$, Derek L.G. Hill ${ }^{1}$, and Nick C. Fox ${ }^{2}$ \\ ${ }^{1}$ Centre for Medical Image Computing (CMIC), Department of Medical Physics and \\ Bioengineering, University College London, London WC1E 6BT, UK \\ o.camara-rey@ucl.ac.uk \\ ${ }^{2}$ Dementia Research Centre (DRC), Institute of Neurology, University College \\ London, Queen Square, London WC1N 3BG, UK
}

\begin{abstract}
The main goal of this work was to assess the accuracy of several well-known methods which provide global (BSI and SIENA) or local (Jacobian integration) estimates of longitudinal atrophy in brain structures using Magnetic Resonance images. For that purpose, we have generated realistic simulated images which mimic the patterns of change obtained from a cohort of 19 real controls and 27 probable Alzheimer's disease patients. SIENA and BSI results correlate very well with gold standard data (BSI mean absolute error $<0.29 \%$; SIENA $<0.44 \%$ ). Jacobian integration was guided by both fluid and FFD-based registration techniques and resulting deformation fields and associated Jacobians were compared, region by region, with gold standard ones. The FFD registration technique provided more satisfactory results than the fluid one. Mean absolute error differences between volume changes given by the FFD-based technique and the gold standard were: sulcal CSF $<2.49 \%$; lateral ventricles $<2.25 \%$; brain $<0.36 \%$; hippocampi $<1.42 \%$.
\end{abstract}

\section{Introduction}

Atrophy measurements in some key brain structures, obtained from structural Magnetic Resonance (MR) images, can be used as biomarkers for neurodegenerative diseases in clinical trials [1, giving complementary information to cognitive tests. Computational anatomy methods [2] have been developed to analyse longitudinal and cross-sectional MR data, including quantification of atrophy.

Until recently, the evaluation of these methods has been extremely difficult since there was no reliable gold standard. Furthermore, semi-automatic or manually traced measurements of regions of interest suffer from lack of reproducibility and sensitivity, as well as being labor-intensive. Recently, Karacali et al. [3] and Camara et al. 4] proposed two different approaches 1 aiming to answer this

${ }^{1}$ Both authors provide tools or gold standard data at the following addresses: https://www.rad.upenn.edu/sbia//http://www.ixi.org.uk 
question. The first technique is based on the generation of topology-preserving deformation fields with Jacobian determinants matching the desired volumetric changes on a specific region of interest. The main drawback of this technique is that it does not take into account the interrelation of different structures. Camara et al. presented a technique in which atrophy in brain structures is simulated with a thermoelastic model of tissue deformation. The main drawback of this method is that it requires a set of segmented structures to build the input of the FEM solver, unlike Karacali's method, which does not necessarily need a segmentation step prior to simulation (the region of interest can be a sphere centered on a manually selected point in the image). On the other hand, in 4], the biomechanical readjustment of structures is modelled, using conventional physics-based techniques based on biomechanical tissue properties.

In this work, we have analysed the accuracy of some well-known longitudinal atrophy measurement methods with a realistic gold standard, providing very valuable information for their use in a clinical context or trials. To the best of our knowledge, this is the first time that such an assessment study, using plausible simulated longitudinal brain atrophy to compare several techniques, has been performed. The gold standard data has been created using an improved version of the methodology presented in [4. Two popular methods, SIENA [5] and BSI [6], that provide global estimates of brain atrophy, and Jacobian integration guided by two different nonrigid registration methods (B-Spline FFD [7] and fluid-based [8] one), that gives local volume changes, were evaluated. A cohort of pairs of MR scans corresponding to 27 probable Alzheimer's Disease (AD) patients and 19 age-matched controls was used to guide the generation of the gold standard data.

\section{Atrophy Simulation}

The methodology for the generation of simulated images involves four main steps: generation of a reference labelled 3D mesh; its adaptation to every subject anatomy; running the FEM solver to simulate regional volumetric change on every subject-specific mesh; and application of the resulting deformation fields to the corresponding baseline MRI.

\subsection{Reference Volumetric Mesh}

A reference tetrahedral mesh (868404 elements) was built using the procedure detailed in 4. In our work, we have employed a different set of labels, according to the information we had from the cohort of real images (both hippocampi and whole brain) and the boundary conditions imposed on the FEM model, as explained in Section 2.3. Therefore, we used labels for the whole brain (Grey Matter and White Matter together), the lateral ventricles, left and right hippocampus, the subtentorial area, extra-sulcal and sulcal cerebrospinal fluid (CSF), the last three being relevant for boundary condition purposes. The separation of the extra-ventricular CSF into two different classes was obtained by applying a brain 


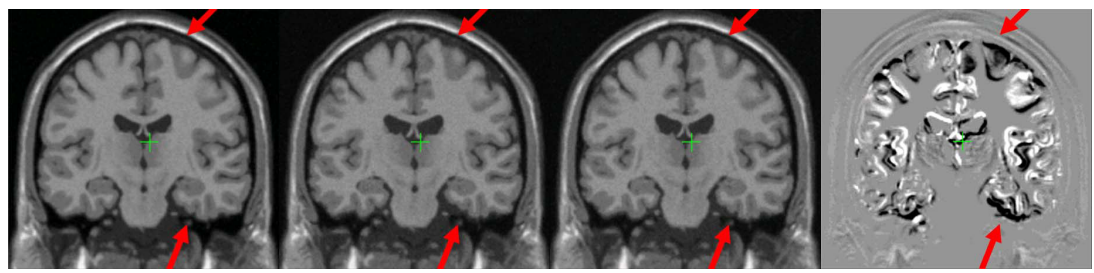

Fig. 1. From left to right: original MNI Brainweb atlas; image with simulated atrophy with old; and new boundary conditions; subtraction between simulated AD images with old and new boundary conditions

cortex segmentation tool, available in Brainvisa 2, on the grey-level version of the MNI Brainweb 3 atlas. The outer interface of the resulting segmentation reaches the brain hull, therefore it includes sulcal CSF, which is isolated using the GM and WM labels of the MNI atlas.

\subsection{Subject-Specific Meshes}

In order to generate a cohort of simulated images with neuroanatomical variation representative of the population, the atlas-based 3D mesh has been adapted to the cohort of real images cited above, thus building a set of corresponding subject-specific meshes, which will be subsequently introduced into the FEM solver. T1-weighted volumetric MR images acquired on a 1.5 Tesla Signa unit (General Electric, Milwaukee) using a 256*256 matrix to provide 124 contiguous $1.5 \mathrm{~mm}$ coronal slices through the head were available for every subject.

The mesh adaptation was performed by applying a Mesh-Warping (MW) technique [9], in which the transformation resulting from a fluid registration [8] between the grey-level atlas image and every subject MR scan is applied to the atlas-based mesh. The classical Jaccard overlap measure of semi-automatically obtained brain-masks [10] after fluid registration was (mean \pm STD) $0.855 \pm 0.024$ for probable ADs and $0.886 \pm 0.018$ for controls, demonstrating a good fitting of the subject-specific meshes.

The fluid registration generates diffeomorphic transformations, thus, theoretically assuring non-negative volume mesh elements after the Mesh-Warping procedure. Nevertheless, we found 8 cases in ADs and 5 in controls with folded elements after MW (mean of 1.62 negative elements in ADs and 1.20 in controls, out of 868404 elements), due to some local Jacobian values very close to zero and the interpolation step needed to warp the meshes. All negative volume elements were corrected by moving conflicting nodes to their neighbour barycentre.

\subsection{Finite-Element Deformation Model}

Model. The subject-specific meshes were introduced into the FEM solver, in which volume changes were simulated with a thermoelastic model of soft tissue

\footnotetext{
2 http://brainvisa.info

3 http://www.bic.mni.mcgill.ca/brainweb/
} 
deformation, based on the TOAST package, which is freely available 4 . After defining the elastic material properties of every region, a set of thermal coefficients, one per structure, that will result in differential regional volume changes to be applied, is computed. These were based on semi-automatically obtained segmentations [10] for both hippocampi and the whole brain between the pairs of MR scans of the cohort described above.

Homogeneous Dirichlet boundary conditions were introduced using a PayneIrons method to suppress the displacement of the mesh nodes corresponding to the surface of the mesh and the subtentorial area. In Camara et al. 4, there was not any restriction on the labels composed of CSF, since they should fill the space left by brain atrophy. This approach could result in unrealistic shifts at the top and the bottom of the brain, as illustrated in Figure 1. We addressed this problem in a different way, by separating the extra-ventricular CSF into sulcal and extra-sulcal CSF, and applying Dirichlet boundary conditions to the latter, notably improving the realism of the simulated images (see Figure 1). This fact raises the question about the behaviour of sulcal and extra-sulcal CSF and their interrelation when atrophy occurs, which is, to the best of our knowledge, not investigated in the literature.

Gold Standard Data. The FEM solution consists of a deformation field, defined at each node of the mesh, which produces the desired volume changes. It can be directly applied to the input mesh or introduced to an interpolation step to generate a voxel-by-voxel defined deformation.

In 4], the volumetric gold standard data was obtained by integrating over every region the volume differences between corresponding elements of the original and warped meshes. Therefore, it did not take into account the interpolation step needed to apply the FEM solution to a grey-level image. In our work, the volumetric gold standard data is obtained directly from the voxel-by-voxel deformation fields after this interpolation step since they are the ones used to generate the simulated grey-level images, i.e. they are a more accurate gold standard. The determinants of the Jacobians of these dense deformation fields were computed and integrated over partial volume Regions of Interest (ROI) defined with available information about the percentages of every tissue on each mesh element. Figure 2 shows an example of a gold standard deformation field and its corresponding Jacobian map for a simulated AD subject.

\section{Atrophy Measurement Techniques}

\subsection{Global Techniques (BSI/SIENA)}

Freeborough and Fox [6] proposed the Boundary Shift Integral (BSI) technique which computes volume change via the amount by which the boundaries of a given cerebral structure have moved. A region around these boundaries is defined with a series of morphological operations, and, subsequently, volume

4 http://www.medphys.ucl.ac.uk/ martins/toast/index.html 


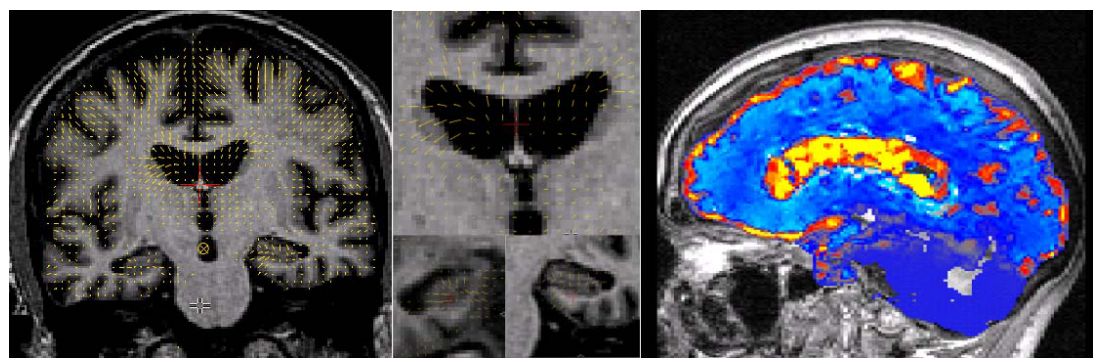

Fig. 2. Gold standard deformation field (left) and zoom into the lateral ventricles (middle, top) and both hippocampi (middle, bottom). The corresponding Jacobian map (right) is also shown (yellow: volume gain; red: volume loss; blue: small volume change).

loss is approximated by integrating the differences in intensities between both MR scans over the defined region, normalized by image intensity means and bounded by predefined upper and lower intensity values. A rigid registration and a semi-automatic segmentation step to delineate the targeted structures are needed as pre-processing steps.

The SIENA (Structural Image Evaluation, using Normalization, of Atrophy) technique, proposed by Smith et al. [5], automatically extracts the brain from a pair of MR images, aligns the brain masked images with an affine transformation constrained by outer skull segmentations and finally estimates atrophy based on the movement of brain edges. The latter step is based on finding all brain surface edges in both MR scans, integrating the distance between the closest matching edge points, multiplied by voxel volume and normalized by the number of points found.

\subsection{Local Techniques (Jacobian Integration)}

The main purpose of Jacobian integration strategies is to capture volume changes within the deformation fields resulting from applying a high-dimensional registration technique between two pairs of MR scans. The analysis of the deformation fields is usually achieved at a voxel-wise level by computing the determinant of their Jacobian matrix, which gives a point-estimate of volumetric change. Additionally, if regions of interest are available, an integration of the Jacobians over these regions gives an estimate of their local volume change. Therefore, the accuracy will depend on the performance of the registration methodology chosen to cope with the deformations between the images. In this work, we have evaluated, for cerebral atrophy measurement purposes, two well-known and widely used registration techniques: B-Spline Free-Form Deformations [7] and a particular implementation of a fluid-based method [8].

The former deforms an image volume by manipulating an underlying mesh of control points. Displacements are then interpolated using the 3D cubic B-spline tensor. For this work, a multi-level FFD was used [11, by first deforming an isotropic FFD mesh of $5 \mathrm{~mm}$ resolution, followed by a further refinement step using a $2.5 \mathrm{~mm}$ resolution mesh. The meshes were adapted to exclude deforma- 


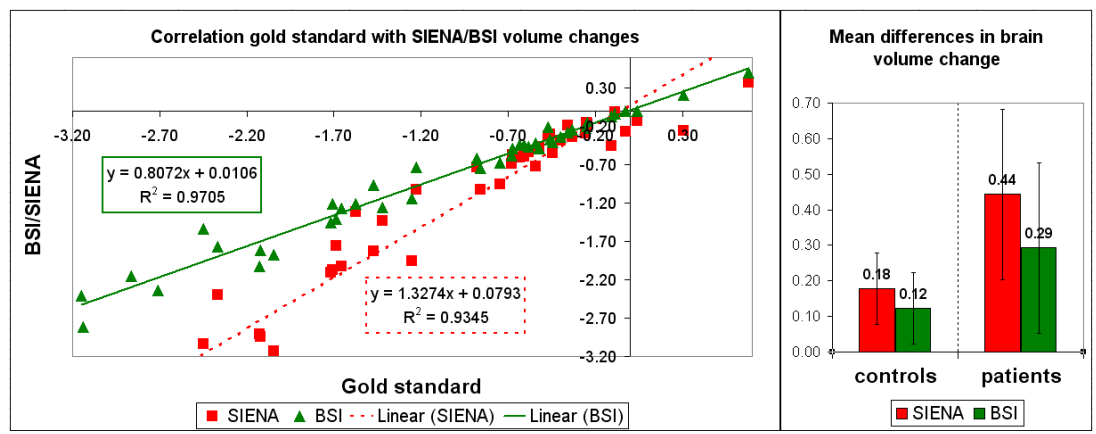

Fig. 3. Correlation (left) and mean differences (right) between gold standard and SIENA/BSI results for simulated controls and patients

tions outside of the reference brain masks. The similarity measure of choice was Normalized Mutual Information. For each level, gradient descent optimization for a maximum of 20 iterations, for 4 steps and initial step sizes of $2 \mathrm{~mm}$ and $1 \mathrm{~mm}$, respectively, was performed.

In fluid registration the transformation is modelled as a viscous flow which warps the source image to match the target image. The driving force was derived from Intensity Cross-Correlation (ICC). The registration was run at half image-resolution, without any masking of structures, for up to 400 iterations subject to the ICC improving globally at each iteration. Further details on the implementation can be found in 8 .

\section{Results}

\subsection{Global Techniques (BSI/SIENA)}

Figure 3 shows a good correlation between the gold standard volume changes and SIENA/BSI results, both for controls and patients. Average absolute differences in brain volume change with respect to the gold standard $(-0.39 \pm 0.74$ for controls and $-1.45 \pm 0.94$ for patients) were small, both for controls (BSI of $0.14 \pm 0.10$; SIENA of $0.18 \pm 0.14$ ) and probable ADs (BSI of $0.29 \pm 0.24$; SIENA of $0.44 \pm 0.48$ ).

SIENA and BSI results provided similar accuracy but their behaviour was different since SIENA tended to overestimate brain volume change whereas BSI underreported atrophy consistently, as illustrated in Figure 3 (left). Another difference can also be observed between the performance of both methods in controls and probable ADs respectively, the latter being more challenging due to a higher amount of brain volume change.

\subsection{Local Techniques (Jacobian Integration)}

Volume change values (mean \pm STD of percentage of the baseline structure volume), for simulated controls and patients, from the gold standard and those provided by the FFD and fluid-based techniques, are shown in Table 1. 
Table 1. Volume changes (mean \pm STD of percentage of the baseline structure volume) provided by gold standard, FFD and fluid techniques

\begin{tabular}{|c||c|c|c||c|c|c||}
\hline \multirow{2}{*}{\multicolumn{1}{|c||}{ Structures }} & \multicolumn{3}{c||}{ Controls } & \multicolumn{3}{c||}{ Patients } \\
\cline { 2 - 7 } & Gold & FFD & Fluid & Gold & FFD & Fluid \\
\hline \hline Extra-sulcal CSF & $1.43 \pm 3.12$ & $0.95 \pm 2.65$ & $0.72 \pm 1.57$ & $4.52 \pm 2.87$ & $3.27 \pm 2.92$ & $1.87 \pm 1.60$ \\
\hline Sulcal CSF & $1.95 \pm 4.20$ & $1.13 \pm 2.50$ & $0.60 \pm 1.32$ & $6.11 \pm 4.07$ & $3.61 \pm 2.94$ & $1.71 \pm 1.48$ \\
\hline Ventricles & $2.24 \pm 5.22$ & $1.96 \pm 4.91$ & $2.04 \pm 4.30$ & $6.02 \pm 4.13$ & $5.09 \pm 3.94$ & $5.08 \pm 3.85$ \\
\hline Left Hippocampi & $-0.03 \pm 1.11$ & $-0.18 \pm 0.56$ & $-0.11 \pm 0.31$ & $-4.00 \pm 2.97$ & $-2.99 \pm 2.13$ & $-1.90 \pm 1.29$ \\
\hline Right Hippocampi & $-0.39 \pm 1.17$ & $-0.35 \pm 0.57$ & $-0.20 \pm 0.54$ & $-4.05 \pm 3.05$ & $-2.80 \pm 2.08$ & $-1.71 \pm 1.33$ \\
\hline Subtentorial Area & $0.09 \pm 0.19$ & $0.03 \pm 0.18$ & $0.09 \pm 0.17$ & $0.30 \pm 0.20$ & $0.23 \pm 0.20$ & $0.28 \pm 0.21$ \\
\hline Whole Brain & $-0.47 \pm 1.01$ & $-0.40 \pm 0.78$ & $-0.32 \pm 0.61$ & $-1.71 \pm 1.12$ & $-1.36 \pm 1.02$ & $-1.06 \pm 0.82$ \\
\hline
\end{tabular}

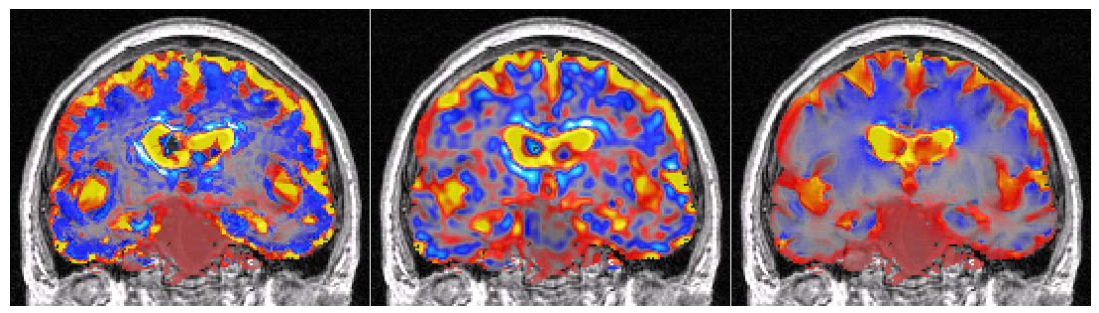

Fig. 4. Jacobian images of gold standard (left) , FFD (centre) and fluid (right) deformation fields (yellow: volume gain; red: volume loss; blue: small volume change)

The FFD-based method provided extremely good accuracy in whole brain and both hippocampi, with larger errors occurring in the sulcal CSF, as was expected due to the complexity of such a region.

The fluid algorithm provided less accurate estimates of volume change for all structures involved in our experiment. An additional difference with respect to the FFD-based technique is the smoothness of the fluid-derived Jacobian map, as shown in Figure 4. This figure allows a visual comparison, for one probable AD case, between the Jacobian maps computed from the gold standard, the FFD and fluid deformation fields. The smoothness of the fluid-based Jacobian map is due to its intrinsic distribution of the volume change in homogeneous regions.

\section{Conclusions}

To the best of our knowledge, this work presents the first report of the accuracy of some well-known atrophy measurement techniques with realistic simulated data. Both global techniques analysed in this paper have been extensively employed for the neuroimaging community and results presented here provide the accuracy obtained by these methods for global volume change estimation purposes. Regarding local methods, the FFD-based one performed better than the fluid registration technique, demonstrating promising results for being used in studies such as clinical trials. Future work will be focused on the definition of good quantitative measures to evaluate the performance of each method and the 
assessment of cross-sectional methods such as Voxel-Based Morphometry ones with realistic simulated data.

Acknowledgement. O. Camara acknowledges support of the EPSRC GR/ S48844/01, Modelling, Understanding and Predicting Structural Brain Change. W.R. Crum acknowledges support of the Medical Images and Signals IRC (EPSRC GR/N14248/01 and UK Medical Research Council Grant No. D2025/31). J.A. Schnabel acknowledges support of the EPSRC GR/S82503/01, Integrated Brain Image Modelling project. R.I. Scahill and N.C. Fox acknowledge support from the UK Medical Research Council, G90/86 and G116/143 respectively.

\section{References}

1. Fox, N., Black, R., Gilman, S., Rossor, M., Griffith, S., Jenkins, L., Koller, M.: Effects of $\mathrm{A} \beta$ immunization (AN1792) on MRI measures of cerebral volume in Alzheimer's disease. Neurology 64, 1563-1572 (2005)

2. Ashburner, J., Csernansky, J., Davatzikos, C., Fox, N., Frisoni, G., Thompson, P.: Computer-assisted imaging to assess brain structure in healthy and diseased brains. Lancet Neurology 2, 79-88 (2003)

3. Karacali, B., Davatzikos, C.: Simulation of tissue atrophy using a topology preserving transformation model. IEEE Transactions on Medical Imaging 25, 649-652 (2006)

4. Camara, O., Schweiger, M., Scahill, R., Crum, W., Sneller, B., Schnabel, J., Ridgway, G., Cash, D., Hill, D., Fox, N.: Phenomenological model of diffuse global and regional atrophy using finite-element methods. IEEE Transactions on Medical Imaging 25, 1417-1430 (2006)

5. Smith, S., Stefano, N.D., Jenkinson, M., Matthews, P.: Normalized accurate measurement of longitudinal brain change. Journal of Computer Assisted Tomography 25(3), 466-475 (2001)

6. Freeborough, P., Fox, N.: The boundary shift integral: an accurate and robust measure of cerebral volume changes from registered repeat MRI. IEEE Transactions on Medical Imaging 16(5), 623-629 (1997)

7. Rueckert, D., Somoda, I., Hayes, C., Hill, D., Leach, M., Hawkes, D.: Nonrigid Registration Using Free-Form Deformations: Applications to Breast MR Images. IEEE Transactions on Medical Imaging 18(8), 712-721 (1999)

8. Crum, W., Tanner, C., Hawkes, D.: Anisotropic multi-scale fluid registration: evaluation in magnetic resonance breast imaging. Physics in Medicine and Biology 50, $5153-5174(2005)$

9. Camara, O., Crum, W., Schnabel, J., Lewis, E., Schweiger, M., Hill, D., Fox, N.: Assessing the quality of Mesh-Warping in normal and abnormal neuroanatomy. In: Medical Image Understanding and Analysis (MIUA 2005), pp. 79-82 (2005)

10. Freeborough, P., Fox, N., Kitney, R.: Interactive algorithms for the segmentation and quantitation of 3-D MRI brain scans. Computer Methods and Programs in Biomedicine 53, 15-25 (1997)

11. Schnabel, J., Tanner, C., Castellano-Smith, A., Leach, M., Hayes, C., Degenhard, A., Hose, R., Hill, D., Hawkes, D.: Validation of Non-Rigid Registration using Finite Element Methods. In: Insana, M.F., Leahy, R.M. (eds.) IPMI 2001. LNCS, vol. 2082, pp. 183-189. Springer, Heidelberg (2001) 